\title{
A Fixed-outline Floorplanning Method Based on 2.5D
}

\author{
Sheqin Dong Qi Xie \\ Department of Computer Science and Technology \\ Tsinghua University, Beijing, 100084, P.R. China
}

\begin{abstract}
In this paper, a fixed-outline floorplanning algorithm based on 2.5D is proposed. By using constraints of area and number of pins to divide the modules into 4 layers, it confines the variations of the widths of the floorplans to a small region through common subsequence of sequence pair representation. The goal of minimizing the area is consistent with that of satisfying the given outline. A set of benchmarks is used for test. Experiments show that the proposed algorithm can achieve high successful probabilities in short run time, even with tight outlines and large aspect ratios are imposed.
\end{abstract}

Keywords: Fixed-outline floorplan, Floorplanning based on 2.5D , Common Subsequence

\section{Intruduction}

Floorplanning is a fundamental problem in the design process of complex chips and has a profound impact on the area, delay, power, and many other design parameters. Many representations for floorplans had been proposed in the last two decades, such as sequence pair [3], O-tree [4], CBL [5], B*tree [6] and BSG [7] etc.

*This work is supported by NSFC 90307005 and NSFC 60473126
Classical floorplanning is formulated as a free-die problem. It roughly determines the layout of a given set of modules subject to various objectives, such as area and estimated total wirelength. In reality, the use of floorplanning during the chip synthesis process almost always comes after the die size and package have been chosen. As pointed in [1], modern floorplanning formulation should be cast as a fixed-outline problem, and the packing must simultaneously achieve zero whitespace and zero overlap for the given outline.

It is also showed in [2] that, empirically, fixed-outline floorplan instances are significantly harder than those without fixed outline. Several works have been done addressed to it. [2] proposed an algorithm based on simulated annealing and sequence pair. It tries to achieve fixed-outline floorplanning by better local search, but the successful probabilities are quite low. [8] presented a genetic algorithm, which can achieve high successful probabilities with loose outlines. But no results of tighter outlines were reported.

On the other hand, interconnect optimization has become a major concern in modern floorplanning. Interconnect delay has dominated over gate delay and timing constraints have become more and more difficult to be met with this huge number of interconnects involved. 2.5D chip is a feasible solution to these problems. It has been shown that interconnect lengths can be greatly reduced in $2.5 \mathrm{D}$ ICs. A $2.5 \mathrm{D}$ 
chip is actually a chip with more than one silicon layers to place modules.

In this paper, a fixed-outline floorplanning algorithm based on $2.5 \mathrm{D}$ is proposed. During simulated annealing, the 2.5D algorithm divides the input modules into 4 layers by adding the constraints of area and number of pins. Then the fixed-outline algorithm is called in each layer. It uses common subsequence and penalty function to bind the variations of the widths of the floorplans to a narrow range. Thus, a simple cost function which has no items about the heights of the floorplans is presented. Experiments show that the proposed algorithm can achieve high successful probabilities in short run time, even with tight outlines and large aspect ratios imposed. Also the proposed algorithm performs well both on $2.5 \mathrm{D}$ and fixed-outline.

\section{Priliminaries}

\subsection{Sequence Pair and Common Sub- sequence}

The concept of sequence pair was first introduced in [3]. A sequence pair is two sequences of all the module names. Horizontal and vertical constraint graphs can be derived from a given sequence pair by the following two rules:

1) $(\ldots a \ldots b \ldots, \ldots a \ldots b \ldots)=>a$ is placed left to $b$;

2) $(\ldots b \ldots a \ldots, \ldots a \ldots b \ldots)=>a$ is placed below to $b$;

Fig.1 gives an example of sequence pair.

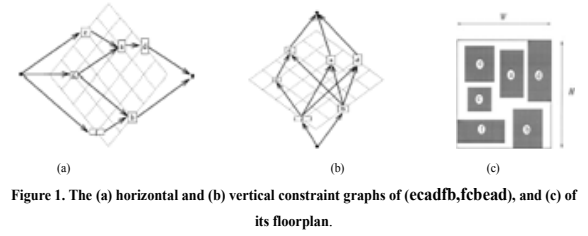

Definition 1. Given two sequences $X$ and $Y$, a sequence $Z$ is a common subsequence of $X$ and $Y$ if $Z$ is a subsequence of both $X$ and $Y$.

For example, assume a sequence pair $\mathrm{P}=($ ecadfb,fcbead $)$, "cad" is a common subsequence of $\mathrm{P}$.

\subsection{Sequence Pair and Common Sub- sequence}

Definition 2. Given a modules set $B$ and an outline with width of $W_{d}$, let $B_{p}=\left\{m_{p 1}, m_{p 2} \ldots m_{p k}\right\}$ be a subset of $B$. If it holds that $\sum_{i=1}^{k} w_{p i} \leq W_{d}$, then $B_{p}$ is a pri-

mary modules set (PMS for short) of $B$. Let $\sum_{i=1}^{k} w_{p i}$ be the length of $B_{p}$ and denote it as $L\left(B_{p}\right)$. Let $\left|B_{p}\right|$ be the number of modules in $B_{p}$.

Definition 3. Given a sequence pair $<\Gamma_{+}, \Gamma_{-}>$and a PMS $B_{p}$, if there exists a common subsequence of $\Gamma_{+}$and $\Gamma_{-}$, which has all the modules in $B_{p}$, we call that $<\Gamma_{+}$, $\Gamma$ > covers $B_{p}$.

For example, assume $B_{p}=\{b, d, e\}$ is a PMS of $\{a, b, c, d, e\}$. Since (bdeac, abdce) contains a common subsequence $\langle b d e\rangle$, which includes all the modules in $B_{p}$, we call that it covers $B_{p}$.

Given above definitions, the following lemma can be drawn:

Lemma 1: If a sequence pair covers a primary modules set $B_{p}$, the width of its floorplan is no less than $L\left(B_{p}\right)$.

\section{The Fixed-outline Algorithm}

The fixed-outline algorithm first randomly creates a sequence pair as the initial solution, and then selects a PMS $B_{p}$ from the given sequence pair. Then during the simulated annealing process, adaptations are performed after perturbations to make all the solutions obtained also cover $B_{p}$. After the adaptations and perturbations, an 
algorithm is called trying to find a better PMS if the fixed-outline constraint has not been satisfied yet. Following are details of the fixed-outline algorithm.

Problem Definition: Each module $m_{i}$ is rectangular and defined by a $\left(w_{i}, h_{i}\right)$, where $w_{i}$ and $h_{i}$ are the width and height of the module respectively. Given a set of modules $B=\left\{m_{1}, m_{2}, \ldots m_{n}\right\}$ and their interconnections, fixed-outline mode floorplanning determines the layout of each module subject to various objectives (such as estimate wirelength, etc.) while satisfying that no modules overlap and it holds that $W_{F} \leq W_{d}$ and $H_{F} \leq H_{d}$, where $W_{d}$ and $H_{d}$ are the width and height of given outline, and $W_{F}$ and $H_{F}$ are those of the floorplan.

For a given set of modules with total area $A_{T}$ and given maximum percent of dead-space $\gamma$, we construct a fixed outline with aspect ratio $\alpha$ by following equations:

$W_{d}=\sqrt{(1+\gamma) A_{T} \alpha} \quad H_{d}=\sqrt{(1+\gamma) A_{T} / \alpha}$

Cost Function: The cost function adopted in the fixed-outline algorithm is:

Cost $=\mathrm{W} 1 *$ area $/$ totalArea $+\mathrm{W} 2 *$ wdiff

Where area is the area of the floorplan, and totalArea is the sum of all the modules' area. While wdiff is defined like this as the penalty function:

wdiff $=$ (width-CurrentCPLength $) /$ CurrentCPLength

Here width is the width of the floorplan, and CurrenCPLength is the length of current common subseqence.

Unlike the cost functions adopted in other algorithms, neither the height of the floorplan nor the excess of the height appear in (1). And the excess of width is only introduced as a penalty. The reason lies in that: since all the solutions obtained cover $B_{p}$, their width must be no less than $\mathrm{L}\left(B_{p}\right)$ (from Lemma 1); by adding penalty function, the widths of solutions are driven exactly to $\mathrm{L}(\mathrm{Bp})$. The criteria of selecting $B_{p}$ (in Section 3.3), guarantees that $L\left(B_{p}\right)$ is very close to $W_{d}$. Thus we regard the width of the solutions as constant, the goal of minimizing the area is consistent with that of minimizing the height. Therefore, it is reasonable to use this simple cost function, which may make the optimization easier.

The Selection of PMS: Firstly, we can get the set $\mathrm{S}$ of all the modules which lie on the right edge of the floorplan using the property of the sequence pair. Then we can find a set of modules from the right edge of the floorplan to the left edge for each module in $\mathrm{S}$. This is a path in the horizontal constraint graphs, also a common sequence of the sequence pair. As we have all the common sequences of the floorplan, it's easy to choose the best one. We only need to compare the length of the common sequences and be sure that it's shorter than the width of the outline.

After the fixed-outline algorithm randomly creates a sequence pair as the initial solution, the algorithm above will be called firstly to generate the initial PMS. If the algorithm fails, we will randomly choose only one module whose length is less than the width of the outline as PMS. Absolutely, such a floorplan won't satisfy the fixed-outline constraint. As pointed before, after the adaptations and perturbations, this algorithm is called trying to find a better PMS if the fixedoutline constraint has not been satisfied yet. Thus, time and time again, we will get the perfect PMS whose length is exactly close to the width of the outline. As we have the reasonable cost function, the fixed-outline constraint will surely be satisfied at last.

Perturbations and Adaptations: The perturbations adopted in the fixed-outline algorithm are the most general ones used in sequence pair representation, which are rotating a module, swapping two modules in the first sequence or in both sequences. Note that in order to maintain the length

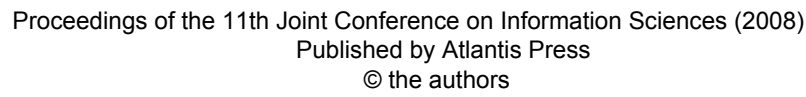


of $B_{p}$, the modules in $B_{p}$ are not allowed to be rotated in our algorithm.

In the following cases, the solution obtained might not cover $B_{p}$ any longer: 1) to swap two modules in the first sequence and only one of them belongs to $B_{p} ; 2$ ) to swap two modules in the first sequence and both of them belong to $B_{p} ; 3$ ) to swap two modules in both sequences and only one of them belongs to $B_{p}$.

To make the solution covers $B_{p}$, adaptations should be taken after perturbations of the above cases. These adaptations can be done in $\mathrm{O}(\mathrm{n})$-time. In the following, we will introduce the adaptation for the first case in detail. The other two kinds of adaptations are quite similar and omitted here for the sake of space.

Let $\left\langle\Gamma_{+}, \Gamma_{-}>\right.$be the sequence pair which already have two modules, $m_{l}$ and $m_{2}\left(m_{l} \in B_{p}, m_{2} \notin B_{p}\right)$, swapped in the first sequence. Let $\Gamma(i)$ denote the $i$-th module in the sequence $\Gamma$.

1) Set sequence $B F=\varnothing$, scan $\Gamma_{\text {. from }}$ left to right, and insert to $B F$ the modules which belong to $B_{p}$. Let $x$ denote the position of $m_{l}$ in $B F$ and $p_{l}$ denote the position of $m_{l}$ in $\Gamma_{-}$;

2) Set $B F=\varnothing$, scan $\Gamma_{+}$from left to right, and insert to $B F$ the modules which belong to $B_{p}$. Let $y$ denote the position of $m_{1}$ in $B F$ and $p_{2}$ denote the position of $m_{l}$ in $\Gamma_{+}$;

3) If $x=y,<\Gamma_{+}, \Gamma_{-}>$already covers $B_{p}$, so the procedure terminates. Otherwise, if $p_{1}<p_{2}$, goto 4; else goto 5;

4) For $i=p_{1}$ to $p_{2}-1$, set $\Gamma_{-}(i)=\Gamma_{-}(i+1)$. Set $\Gamma_{-}\left(p_{2}\right)=m_{l}$ and stop;

For $i=p_{2}+1$ to $p_{1}$, set $\Gamma_{-}(i)=\Gamma_{-}(i-1)$. Set $\Gamma_{\text {. }}$ $\left(p_{2}\right)=m_{1}$ and stop;

\section{The Algorithm Based on 2.5D}

Before using the fixed-outline algorithm, we need an algorithm to group modules.
We set the numbers of layers to 4 . The details will be discussed next.

Constraint: We choose two constraints for our 2.5D algorithm. They are constraints of total area and total number of pins. As in the fixed-outline algorithm, we construct a fixed outline by using the total area of modules. So to be sure that each layer has the same fixedoutline, the total area of each layer should be more or less the same. We also choose the total numbers of pins. The $2.5 \mathrm{D}$ algorithm is firstly designed to decrease the interconnect lengths by adding interconnects of layers. In theory, the interconnects of layers will be bigger if the number of pins in each layer is more approximate.

Cost Function: We set our cost function as this:

Cost $=|\operatorname{ADiff}(0)|+\ldots+|\operatorname{ADiff}(3)|+|\operatorname{PDiff}(0)|$

$+\ldots+|\operatorname{Diff}(3)|$ 。

Where ADiff(i) means the diff between total area of layer $i$ and quarter of total area of all the modules in 4 layers. Similarly, PDiff(i) covers the diff of number of pins. And we treat the area constraint and the pins constraint equally. The cost function is simple but effective.

Perturbations: We generate the initial solution randomly. We choose the two layers whose total area diff most. Then randomly choose two modules for swap. If after the swap, the diff between two layers can be reduced by 10 percents at least, we will accept it. Otherwise, we have to choose another two.

\section{Experimental Results}

We implemented the proposed algorithm in $\mathrm{C}++$ and run test data created from MCNC benchmarks on a PC with AMD Athlon ${ }^{\mathrm{TM}} 64$ Processor 3000+ 1.81GHZ and 1.00GB RAM. The following experimental results are the averages 
of 100 runs. Note that the modules handled in our experiments are all hard modules.

Table 1 shows that the proposed algorithm are quite promising in both successful probabilities and run time, even when the aspect ratio is as large as 6 . Another advantage of the proposed algorithm is that high successful probabilities can be achieved with tight outline imposed. When $\gamma=7 \%$ and $6 \%$, the successful probabilities is $100 \%$. When $\gamma=5 \%$, the successful probabilities range from $70 \%$ to $90 \%$, which are still acceptable. Fig.2, 3 and 4 shows several resultant floorplans of 198(created from ami33) with $\gamma=7 \%$ and aspect ratios of 1 , 2 and 6 respectively. In the above experiments, the algorithm terminates once a feasible floorplan. Otherwise, we regard the algorithm failed.

\section{Conclusions}

In this paper, a fixed-outline floorplanning algorithm based on 2.5D is proposed. The fixed-outline floorplanning algorithm uses common subsequence of sequence pair. It tries to confine the variations of the widths of the floorplans to a small region through common subsequence. Thus the goal of minimizing the area is consistent with that of satisfying the given outline. While the $2.5 \mathrm{D}$ algorithm uses the concept of layers. SA is used by adding constraints of area and number of pins to divide the modules into 4 layers. A lot of standard benchmark files are used for testing. The results are promising.

\section{References}

[1] A.B. Kahng, "Classical Floorplanning Harmful?" Proceedings of ISPD, San Diego, 2000, pp. 207-213.

[2] Saurabh N. Adya, Igor L. Markov, "Fixed-outline Floorplanning Through Better Local Search", Proceedings of ICCD, Austin, 2001, pp. 328-334.

[3] H. Murata, K. Fujiyoshi, S. Nakatake et.al, "VLSI module placement based on rectangle-packing by the sequence pair", IEEE Trans. on CAD, 1996, vol. 15(12), pp. 1518-1524.

[4] Y. Pang, C.-K. Cheng and T. Yoshimura, "An Enhanced Perturbing Algorithm for Floorplan Design Using the O-tree Representation", Proceedings of ISPD, 2000, pp. 168-173.

[5] Xianlong Hong et al., "Corner Block List: An Effective and Efficient Topological Representation of onSlicing Floorplan", Proceedings of ICCAD, 2000, pp. 8-13.

[6] Y.-C. Chang, Y.-W. Chang, G.-M. $\mathrm{Wu}$ and S.-W. Wu, "B*-Trees: A New Representation for Non-Slicing Floorplans", Proceedings of $D A C$, 2000, pp. 458-463.

[7] S. Nakatake, K. Fujiyoushi, H. Murata and Y. Kajitani, "Module Placement on BSG-structure and IC Layout Applications," Proceedings of $I C$ CAD, 2000, pp. 8-13.

[8] Chang-Tzu Lin, De-Sheng Chen et al, "Robust Fixed-outline Floorplanning Through Evolutionary Search," Proceedings of $A S P-D A C, 2004$, pp 4244.

Table 1. The results of 198 with $\gamma=7 \%$

\begin{tabular}{|c|c|c|c|c|c|c|}
\hline Aspect ratio & $\boldsymbol{\alpha}=\mathbf{1}$ & $\boldsymbol{\alpha}=\mathbf{2}$ & $\boldsymbol{\alpha}=\mathbf{3}$ & $\boldsymbol{\alpha}=\mathbf{4}$ & $\boldsymbol{\alpha}=\mathbf{5}$ & $\boldsymbol{\alpha}=\mathbf{6}$ \\
\hline Run time & $4.9 \mathrm{~s}$ & $3.1 \mathrm{~s}$ & $3.7 \mathrm{~s}$ & $3.3 \mathrm{~s}$ & $4.1 \mathrm{~s}$ & $4.9 \mathrm{~s}$ \\
\hline Successful & $100 \%$ & $100 \%$ & $100 \%$ & $100 \%$ & $100 \%$ & $100 \%$ \\
\hline
\end{tabular}

\footnotetext{
Proceedings of the 11th Joint Conference on Information Sciences (2008)

Published by Atlantis Press (C) the authors
} 

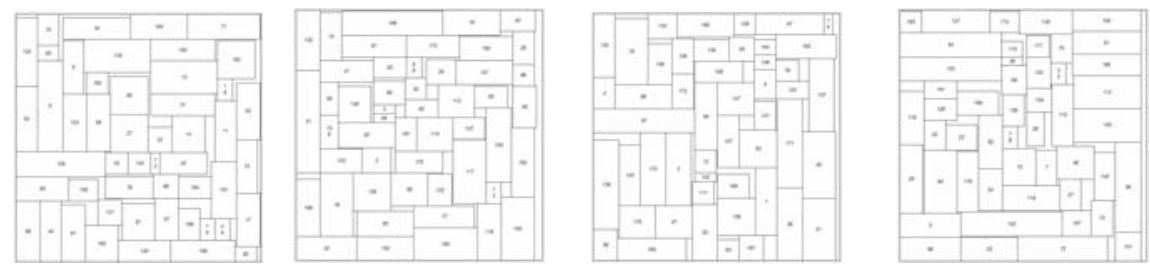

Figure 2. The resultant floorplans of 198 in 4 layers with $\gamma=7 \%$ and aspect ratios of 1 .
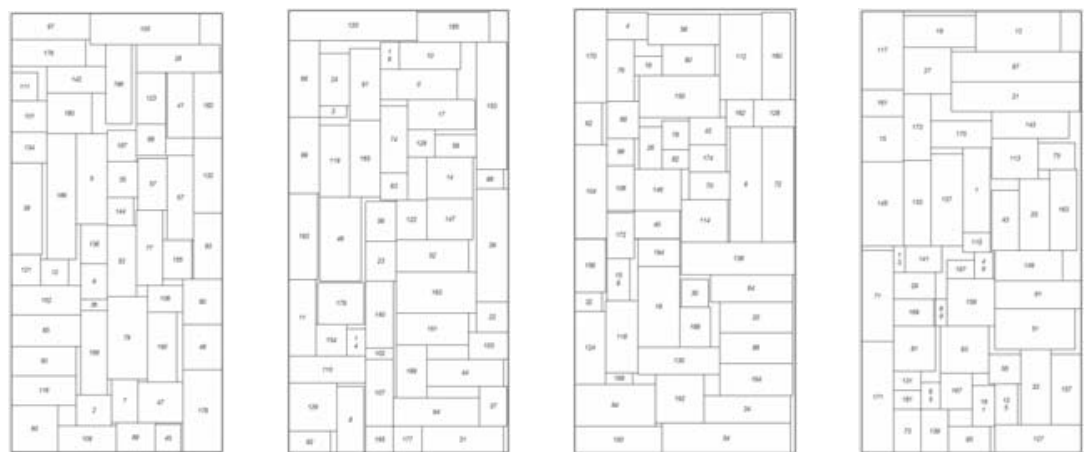

Figure 3. The resultant floorplans of 198 in 4 layers with $\gamma=7 \%$ and aspect ratios of 2.
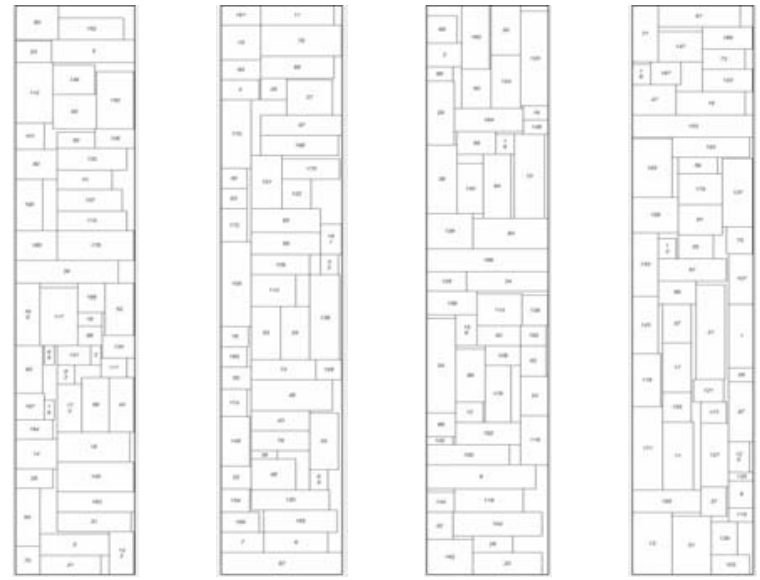

Figure 4. The resultant floorplans of 198 in 4 layers with $\gamma=7 \%$ and aspect ratios of 6 .

Proceedings of the 11th Joint Conference on Information Sciences (2008)

Published by Atlantis Press (c) the authors 